\title{
All optical binary ASK demodulator using photonic crystal based nonlinear ring resonator
}

\author{
MAHSA NARIMANZADEH, AliREZA ANDALIB* \\ Department of Electrical Engineering, Tabriz Branch, Islamic Azad University, Tabriz, Iran \\ *Corresponding author: andalib@iaut.ac.ir
}

\begin{abstract}
In this paper we will propose and design an all optical amplitude shift keying demodulator using a photonic crystal based nonlinear ring resonator. The proposed structure will be designed such that it can generate digital 0 and 1 based on the amplitude of the input signal. The working mechanism is based on controlling the optical behavior of the resonant ring using optical intensity. The proposed structure was simulated using plane wave expansion and finite difference time domain methods. The simulation results show that the rise and fall times for the proposed structure are 0.5 and $0.1 \mathrm{ps}$, respectively.
\end{abstract}

Keywords: photonic crystal, amplitude shift keying (ASK), optical demodulator, delay time.

\section{Introduction}

Amplitude shift keying (ASK) is a form of amplitude modulation which is used for representing digital data based on variation of signal amplitude. Optical modulation is a promising way to transmit low frequency digital data using high frequency carrier waves. The simplest way of using ASK modulation is to choose two different wave amplitudes for digital 1 and 0 . After receiving the modulated signal, one needs an all optical demodulator to convert the received waveform into a binary code.

Due to their high band width and high speed, optical waves are very useful for transmitting data and can be used as excellent carrier waves in different modulation techniques. For working with optical waves one needs optical devices, whose inputs, outputs and processing parts should be purely optical.

In last decades it has been shown that photonic crystals (PhCs) $[\underline{1}, \underline{2}]$ which consist of regular arrangement of dielectric materials with periodic refractive indices can be very useful for designing different kinds of optical devices [ $\underline{3}-\underline{5}]$. The realization of optical filters [ $\underline{6}-\underline{10}]$, demultiplexers $[\underline{11}-\underline{16}]$, logic gates $[\underline{17}-\underline{22}]$, coders $[\underline{23}-\underline{29}]$, adders [ $\underline{30}-\underline{35}]$ and analog to digital converters [은 $\underline{40}$ ] proves the ability of photonic crystals for playing a crucial role in the next generation of all optical networks. 
An all optical phase shift keying (PSK) demodulator was designed using 2D PhCs [41]]. The proposed structure can produce binary 0 and 1 codes considering the phase of the received optical signal. The proposed structure works base on constructive and destructive interference of the input signal with the reference signal. Besides this PSK modulator, some methods also have been proposed for designing all optical analog to digital converters $[\underline{39}, \underline{42}, \underline{43}]$. But no works have been proposed for designing ASK modulators using $\mathrm{PhCs}$.

In this paper we are going to design an all optical binary ASK demodulator. The proposed structure works based on threshold switching principle [44, 45]. In the final structure, two binary 0 and 1 codes can be produced based on the amplitude of an input signal.

\section{Design procedure}

The basic structure used for designing the proposed structure consists of periodic $2 \mathrm{D}$ array of dielectric rods with square lattice. The radius and refractive index of the dielectric rods are $122 \mathrm{~nm}$ and 3.46, respectively. The lattice constant of the basic structure is $581 \mathrm{~nm}$. As shown in Fig. 1, the main photonic band gap of the fundamental structure is at $0.28-0.42$ normalized frequencies, which is equal to $1450-1210 \mathrm{~nm}$ wavelength range.

The proposed ASK demodulator was designed using a nonlinear ring resonator. The nonlinear ring consists of 3 main parts, a $5 \times 5$ square shaped core, 24 nonlinear rods and the outer shell of the ring. The nonlinear rods are of doped glass whose linear refractive index and the Kerr coefficient are 1.4 and $10^{-14} \mathrm{~m}^{2} / \mathrm{W}$ [31]. Two orthogonal waveguides were created near the resonant ring. These waveguides were named BUS and DROP. The front and end side of the BUS were labeled as B and O. The third waveguide was created some rows below the BUS which connects I to the resonant ring.

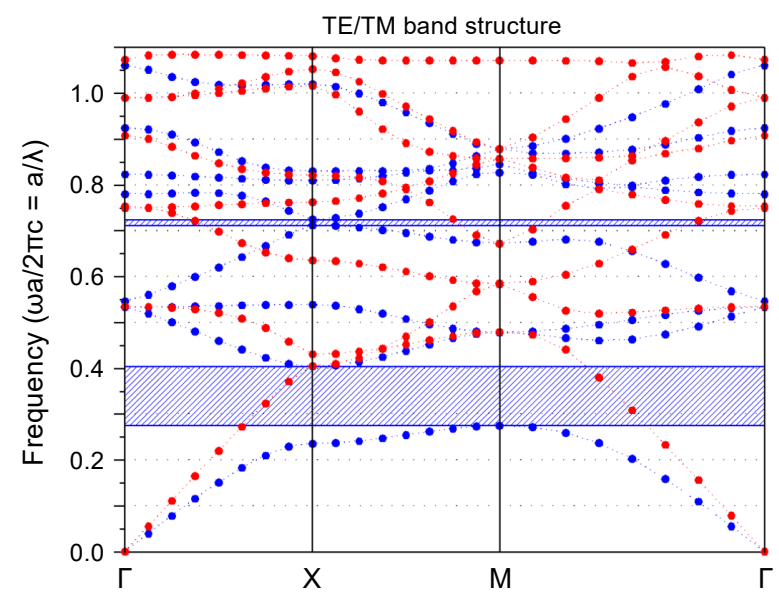

Fig. 1. The band structure diagram of the basic $\mathrm{PhC}$. 


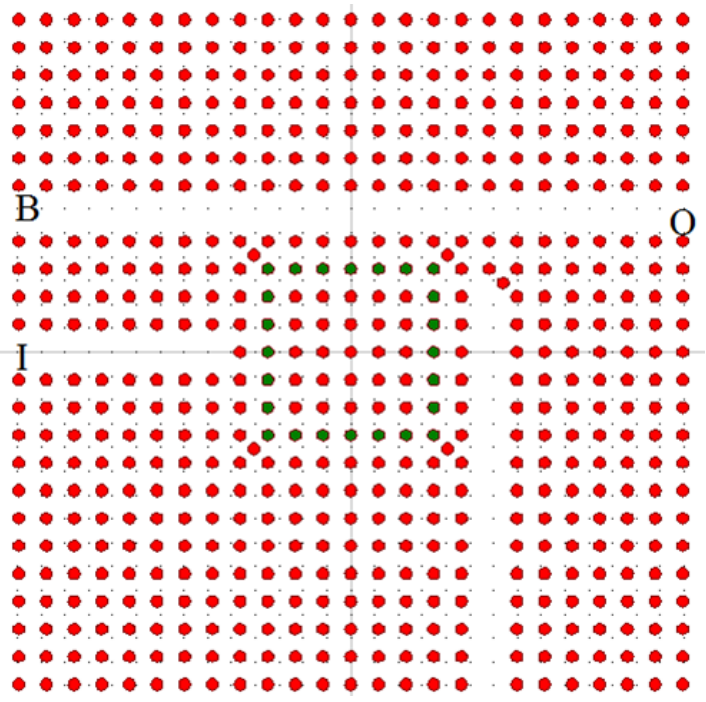

Fig. 2. The proposed structure for optical ASK demodulator.

The final structure is shown in Fig. 2, in which B, I and $\mathrm{O}$ are bias, input and output ports, respectively.

\section{Simulation and results}

The spectrum diagram of the nonlinear resonant ring at low optical intensities is shown in Fig. 3. As depicted in Fig. 3, the nonlinear resonant ring has a resonant mode at $1560 \mathrm{~nm}$. Also Fig. 4 shows the output spectra for three different values of the refrac-

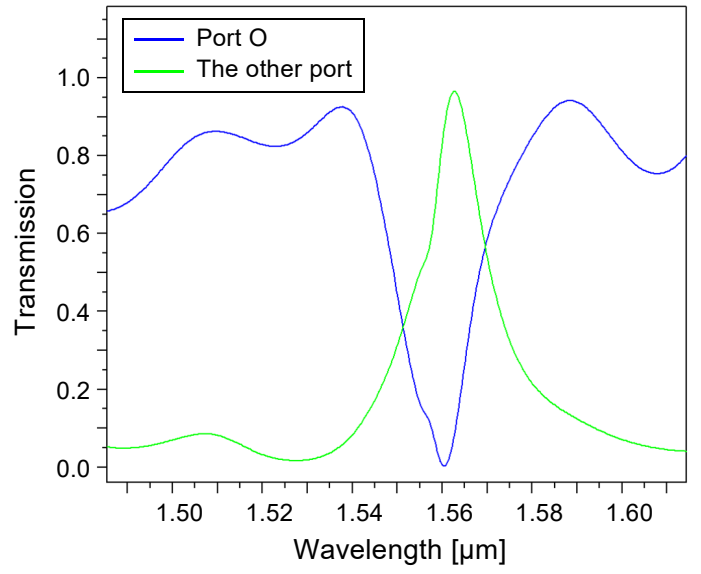

Fig. 3. The wavelength spectrum of the resonant ring. 


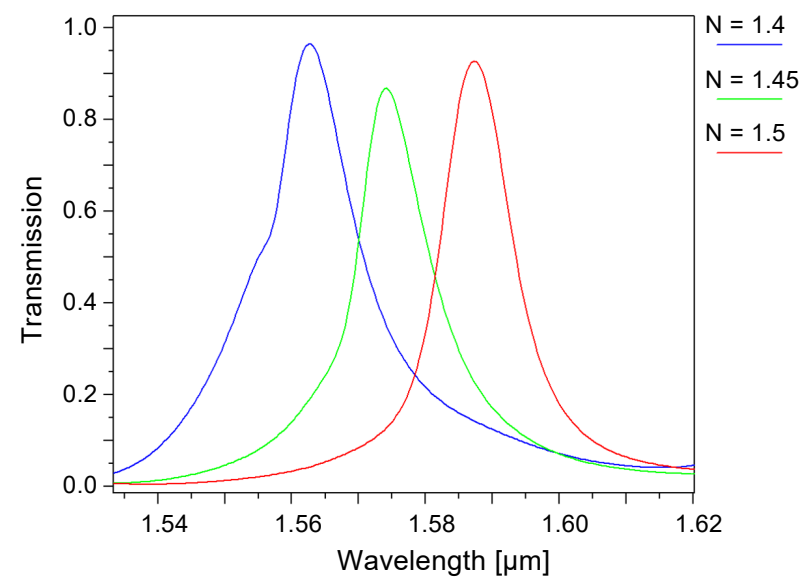

Fig. 4. The output spectra of the resonant ring for different values of the refractive index of nonlinear rods.

tive index of nonlinear rods, which shows that increasing the refractive index will shift the resonant mode ring toward upper wavelengths. This proves that one can control the optical behavior of the resonant ring using high optical intensities.

For simulating the final structure, we used two optical Gaussian sources at B and I ports. Both has the same wavelength which is equal to $1560 \mathrm{~nm}$. The optical intensity of $\mathrm{B}$ is constant and equal to $1 \mathrm{~W} / \mu \mathrm{m}^{2}$. Two different optical intensities were used at $\mathrm{I}$. When the optical intensity at I is about $0.2 \mathrm{~W} / \mu \mathrm{m}^{2}$, the optical intensity near the resonant ring is smaller than the optical intensity required for triggering the nonlinear effects and shifting the resonant mode, therefore the resonant ring can drop the optical waves from BUS into DROP. As a result, no optical waves can reach the output port, so it remains $\mathrm{OFF}$ (i.e. logic 0 ). However when the optical intensity at $\mathrm{I}$ is about $1 \mathrm{~W} / \mu \mathrm{m}^{2}$,

a
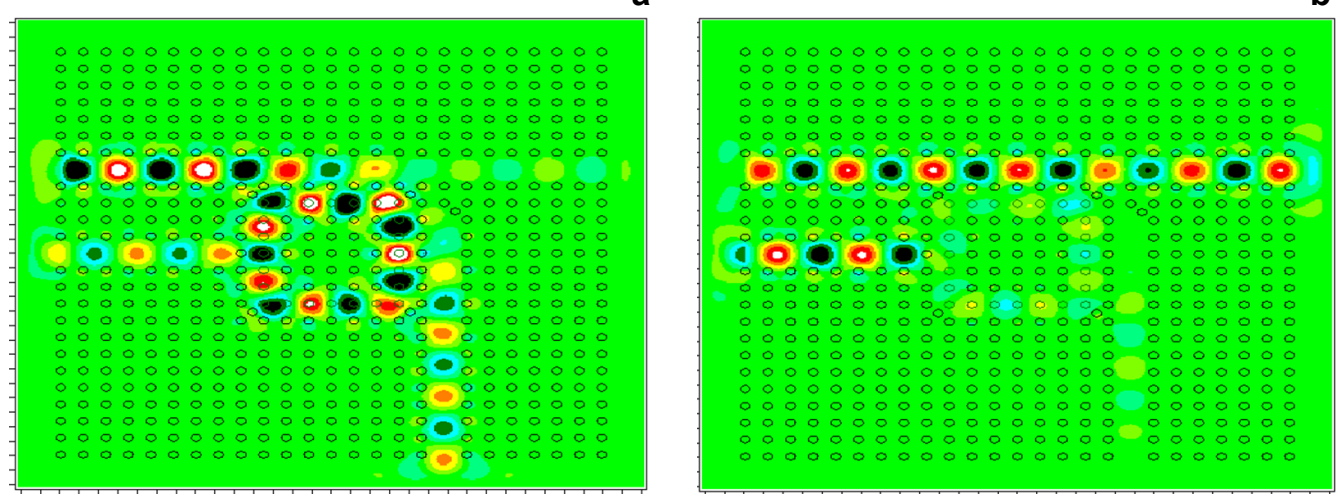

Fig. 5. The optical behavior of the optical ASK demodulator when the input intensity is $0.2 \mathrm{~W} / \mu \mathrm{m}^{2}$ (a), and $1 \mathrm{~W} / \mu \mathrm{m}^{2}(\mathbf{b})$. 

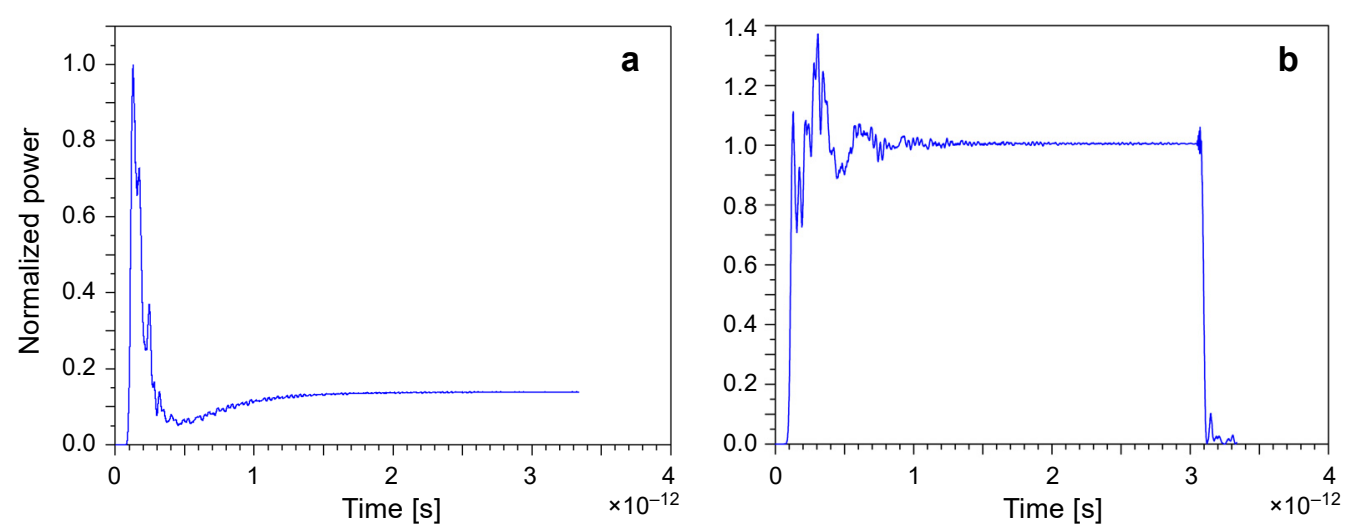

Fig. 6. Time response diagrams of the optical ASK demodulator when the input intensity is $0.2 \mathrm{~W} / \mu \mathrm{m}^{2}$ (a), and $1 \mathrm{~W} / \mu \mathrm{m}^{2}(\mathbf{b})$.

this amount of optical intensity can change the resonant mode and trigger the threshold switching mechanism. Therefore the resonant ring cannot drop the optical waves from BUS into DROP. As a result, the optical waves can reach the output port and turn it ON (i.e. logic 1). These procedures are shown in Fig. 5.

The time response diagrams of the proposed structure for both working states are shown in Fig. 6. When the optical intensity at I is about $0.2 \mathrm{~W} / \mu \mathrm{m}^{2}$, the amount of normalized optical intensity at the $\mathrm{O}$ is about $10 \%$. On the other hand, when the optical intensity at $\mathrm{I}$ is about $1 \mathrm{~W} / \mu \mathrm{m}^{2}$, the amount of normalized optical intensity at $\mathrm{O}$ is about $100 \%$. The rise and fall times for the proposed structure are 0.5 and $0.1 \mathrm{ps,}$, respectively.

\section{Conclusion}

A novel structure was proposed for implementing an all optical binary ASK demodulator using a PhCs-based nonlinear ring resonator. The proposed structure works based on controlling the optical behavior of the resonant ring using optical intensity. When the optical intensity is about $0.2 \mathrm{~W} / \mu \mathrm{m}^{2}$, the demodulator generates binary 0 at the output port, however when the input optical intensity is about $1 \mathrm{~W} / \mu \mathrm{m}^{2}$, the demodulator generates binary 1 at the output port. The rise and fall times for the proposed structure are 0.5 and $0.1 \mathrm{ps}$, respectively.

\section{References}

[1] JoHn S., Strong localization of photons in certain disordered dielectric superlattices, Physical Review Letters 58(23), 1987, pp. 2486-2489, DOI: 10.1103/PhysRevLett.58.2486.

[2] Yablonovitch E., Inhibited spontaneous emission in solid-state physics and electronics, Physical Review Letters 58(20), 1987, pp. 2059-2062, DOI: 10.1103/PhysRevLett.58.2059.

[3] Liu D., Gao Y., Tong A., Hu S., Absolute photonic band gap in 2D honeycomb annular photonic crystals, Physics Letters A 379(3), 2015, pp. 214-217, DOI: 10.1016/j.physleta.2014.11.030. 
[4] Diaz-Valencia B.F., Calero J.M., Photonic band gaps of a two-dimensional square lattice composed by superconducting hollow rods, Physica C: Superconductivity and its Applications 505, 2014, pp. 74-79, DOI: 10.1016/j.physc.2014.07.012.

[5] Wang Z., Gao M., Ullah Z., Chen H., Zhang D., Zhang Y., Zhang Y., Optical processes in different types of photonic band gap structures, Optical Materials 44, 2015, pp. 58-66, DOI: 10.1016/j.optmat. 2015.03.001.

[6] Moloudian G., Sabbaghi-Nadooshan R., Hassangholizadeh-Kashtiban M., Design of all-optical tunable filter based on two-dimensional photonic crystals for WDM (wave division multiplexing) applications, Journal of the Chinese Institute of Engineers 39(8), 2016, pp. 971-976, DOI: 10.1080/ 02533839.2016.1215937.

[7] Dideban A., Habibiyan H., Ghafoorifard H., Photonic crystal channel drop filter based on ring -shaped defects for DWDM systems, Physica E: Low-dimensional Systems and Nanostructures 87, 2017, pp. 77-83, DOI: 10.1016/j.physe.2016.11.022.

[8] Tavousi A., Mansouri-Birjandi M.A., Ghadrdan M., Ranjbar-Torkamani M., Application of photonic crystal ring resonator nonlinear response for full-optical tunable add-drop filtering, Photonic Network Communications 34, 2017, pp. 131-139, DOI: 10.1007/s11107-016-0680-x.

[9] Alipour-Banaei H., Serajmohammadi S., Mehdizadeh F., Hassangholizadeh-Kashtiban M., Special optical communication filter based on Thue-Morse photonic crystal structure, Optica Applicata 46(1), 2016, pp. 145-152, DOI: 10.5277/oa160113.

[10] Wang Y., Chen D., Zhang G., Wang J., TAo S., A super narrow band filter based on silicon 2D photonic crystal resonator and reflectors, Optics Communications 363, 2016, pp. 13-20, DOI: 10.1016/ j.optcom.2015.10.070.

[11] Talebzadeh R., Soroosh M., Mehdizadeh F., Improved low channel spacing high quality factor four-channel demultiplexer based on photonic crystal ring resonators, Optica Applicata 46(4), 2016, pp. 553-564, DOI: 10.5277/oa160404.

[12] Balaji V.R., Murugan M., Robinson S., Optimization of DWDM demultiplexer using regression analysis, Journal of Nanomaterials, Vol. 2016, (2016), article 9850457, DOI: 10.1155/2016/9850457.

[13] Kannaiyan V., Savarimuthu R., Dhamodharan S.K., Investigation of 2D-photonic crystal resonant cavity based WDM demultiplexer, Opto-Electronics Review 26(2), 2018, pp. 108-115, DOI: 10.1016/ j.opelre.2018.01.002.

[14] Kannaiyan V., Savarimuthu R., Dhamodharan S.K., Performance analysis of an eight channel demultiplexer using a 2D-photonic crystal quasi square ring resonator, Opto-Electronics Review 25(2), 2017, pp. 74-79, DOI: 10.1016/j.opelre.2017.05.003.

[15] RafieE E., Emami F., Design of a novel all-optical ring shaped demultiplexer based on two-dimensional photonic crystals, Optik 140, 2017, pp. 873-877, DOI: 10.1016/j.ijleo.2017.05.010.

[16] Mehdizadeh F., Soroosh M., Alipour-Banaei H., An optical demultiplexer based on photonic crystal ring resonators, Optik 127(20), 2016, pp. 8706-8709, DOI: 10.1016/j.ijleo.2016.06.086.

[17] Alipour-Banaei H., Serajmohammadi S., Mehdizadeh F., All optical Nand gate based on nonlinear photonic crystal ring resonators, Optik 130, 2017, pp. 1214-1221, DOI: 10.1016/j.ijleo.2016.11.190.

[18] Karimzadeh M., Andalib A., A proposal for all optical XNOR gate using photonic crystal based nonlinear cavities, Journal of Optical Communications, 2018, DOI: 10.1515/joc-2018-0089.

[19] Kumar A., Gupta M.M., MedheKar S., All-optical NOT and AND gates based on 2D nonlinear photonic crystal ring resonant cavity, Optik 167, 2018, pp. 164-169, DOI: 10.1016/j.ijleo.2018.04.042.

[20] Areed N.F.F., El Fakharany A., Hameed M.F.O., Obayya S.S.A., Controlled optical photonic crystal AND gate using nematic liquid crystal layers, Optical and Quantum Electronics 49, 2017, article 45, DOI: $10.1007 / \mathrm{s} 11082-016-0852-\mathrm{z}$.

[21] MoniEM T.A., All-optical XNOR gate based on 2D photonic-crystal ring resonators, Quantum Electronics 47(2), 2017, p. 169, DOI: 10.1070/QEL16279.

[22] Mehdizadeh F., Soroosh M., Designing of all optical NOR gate based on photonic crystal, Indian Journal of Pure and Applied Physics 54(1), 2016, pp. 35-39. 
[23] Salimzadeh S., Alipour-Banaei H., A novel proposal for all optical 3 to 8 decoder based on nonlinear ring resonators, Journal of Modern Optics 65(17), 2018, pp. 2017-2024, DOI: $\underline{10.1080 / 09500340 .}$ 2018.1489077.

[24] Daghooghi T., Soroosh M., Ansari-Asl K., Ultra-fast all-optical decoder based on nonlinear photonic crystal ring resonators, Applied Optics 57(9), 2018, pp. 2250-2257, DOI: 10.1364/AO.57.002250.

[25] Mehdizadeh F., Alipour-Banaei H., Serajmohammadi S., Design and simulation of all optical decoder based on nonlinear PhCRRs, Optik 156, 2018, pp. 701-706, DOI: 10.1016/j.ijleo.2017.12.011.

[26] Moniem T.A., All optical active high decoder using integrated 2D square lattice photonic crystals, Journal of Modern Optics 62(19), 2015, pp. 1643-1649, DOI: 10.1080/09500340.2015.1061061.

[27] Mendizadeh F., Alipour-Banaei H., Serajmohammadi S., Study the role of non-linear resonant cavsities in photonic crystal-based decoder switches, Journal of Modern Optics 64(13), 2017, pp. 1233-1239, DOI: $\underline{\text { 10.1080/09500340.2016.1275854. }}$

[28] Mehdizadeh F., Soroosh M., Alipour-Banaei H., Proposal for 4-to-2 optical encoder based on photonic crystals, IET Optoelectronics 11(), 2017, pp. 29-35, DOI: 10.1049/iet-opt.2016.0022.

[29] Seif-Dargahi H., Ultra-fast all-optical encoder using photonic crystal-based ring resonators, Photonic Network Communications 36, 2018, pp. 272-277, DOI: 10.1007/s11107-018-0779-3.

[30] Serajmohammadi S., Alipour-Banaei H., Mehdizadeh F., Proposal for realizing an all-optical half adder based on photonic crystals, Applied Optics 57(7), 2018, pp. 1617-1621, DOI: 10.1364/ AO.57.001617.

[31] Rahmani A., Mehdizadeh F., Application of nonlinear PhCRRs in realizing all optical half-adder, Optical and Quantum Electronics 50, 2018, article 30, DOI: 10.1007/s11082-017-1301-3.

[32] Andalib A., A novel proposal for all-optical Galois field adder based on photonic crystals, Photonic Network Communications 35, 2018, pp. 392-396, DOI: 10.1007/s11107-017-0756-2.

[33] JiAng Y.-C., LiU S.-B., Zhang H.-F., Kong X.-K., Realization of all optical half-adder based on self -collimated beams by two-dimensional photonic crystals, Optics Communications 348, 2015, pp. 90 -94, DOI: 10.1016/j.optcom.2015.03.011.

[34] Jae Hun Kim, Young Tae Byun, Young Min Jhon, Seok Lee, Deok Ha Woo, Sun Ho Kim, All-optical half adder using semiconductor optical amplifier based devices, Optics Communications 218(4-6), 2003, pp. 345-349, DOI: 10.1016/S0030-4018(03)01203-3.

[35] KarkhanehChi M.M., Parandin F., Zahedi A., Design of an all optical half-adder based on 2D photonic crystals, Photonic Network Communications 33, 2017, pp. 159-165, DOI: 10.1007/s11107-016-0629-0.

[36] Mehdizadeh F., Soroosh M., Alipour-Banaei H., Farshidi E., All optical 2-bit analog to digital converter using photonic crystal based cavities, Optical and Quantum Electronics 49, 2017, article 38, DOI: $10.1007 / \mathrm{s} 11082-016-0880-8$.

[37] Mehdizadeh F., Soroosh M., Alipour-Banaei H., Farshidi E., A novel proposal for all optical analog-to-digital converter based on photonic crystal structures, IEEE Photonics Journal 9(2), 2017, pp. 1-11, DOI: 10.1109/JPHOT.2017.2690362.

[38] Mehdizadeh F., Soroosh M., Alipour-Banaei H., Farshidi E., Ultra-fast analog-to-digital converter based on a nonlinear triplexer and an optical coder with a photonic crystal structure, Applied Optics 56(7), 2017, pp. 1799-1806, DOI: 10.1364/AO.56.001799.

[39] FASIHI K., All-optical analog-to-digital converters based on cascaded 3-dB power splitters in 2D photonic crystals, Optik 125(21), 2014, pp. 6520-6523, DOI: 10.1016/j.ijleo.2014.08.030.

[40] Konishi T., Tanimura K., Asano K., Oshita Y., Ichioka Y., All-optical analog-to-digital converter by use of self-frequency shifting in fiber and a pulse-shaping technique, Journal of the Optical Society of America B 19(11), 2002, pp. 2817-2823, DOI: 10.1364/JOSAB.19.002817.

[41] Karimzadeh M., Andalib A., All optical BPSK demodulator using photonic crystal based coupled waveguides, Journal of Optical Communications, 2018, DOI: 10.1515/joc-2018-0060.

[42] Youssefi B., Moravvej-Farshi M.K., Granpayeh N., Two bit all-optical analog-to-digital converter based on nonlinear Kerr effect in 2D photonic crystals, Optics Communications 285(13-14), 2012, pp. 3228-3233, DOI: 10.1016/j.optcom.2012.02.081. 
[43] Jafari D., Nurmohammadi T., Asadi M.J., Abbasian K., All-optical analog-to-digital converter based on Kerr effect in photonic crystal, Optics and Laser Technology 101, 2018, pp. 138-143, DOI: 10.1016/j.optlastec.2017.11.007.

[44] Afzal S., Ahmadi V., Ebnali-Heidari M., All-optical tunable photonic crystal nor gate based on the nonlinear Kerr effect in a silicon nanocavity, Journal of the Optical Society of America B 30(9), 2013, pp. 2535-2539, DOI: 10.1364/JOSAB.30.002535.

[45] Danaie M., KaAtuZian H., Design and simulation of an all-optical photonic crystal AND gate using nonlinear Kerr effect, Optical and Quantum Electronics 44, 2012, pp. 27-34, DOI: 10.1007/s11082 $\underline{-011-9527-\mathrm{y} .}$

Received November 26, 2018 in revised form February 9, 2019 